\title{
Chemokine profiling in serum from patients with ovarian cancer reveals candidate biomarkers for recurrence and immune infiltration
}

\author{
AGATA MLYNSKA $^{1,2}$, GRETA SALCIUNIENE ${ }^{1}$, KAROLINA ZILIONYTE $^{1}$, SIMA GARBERYTE ${ }^{1}$, \\ MARIUS STRIOGA $^{1}$, BIRUTE INTAITE ${ }^{1}$, AUSRINE BARAKAUSKIENE ${ }^{2}$, \\ GIANROCCO LAZZARI ${ }^{3}$, NERINGA DOBROVOLSKIENE ${ }^{1}$, \\ JAN ALEKSANDER KRASKO $^{1}$ and VITA PASUKONIENE ${ }^{1}$ \\ ${ }^{1}$ Laboratory of Immunology, National Cancer Institute, Vilnius LT-08660; ${ }^{2}$ Life Sciences Center, Vilnius University, \\ Vilnius LT-10257, Lithuania; ${ }^{3}$ Swiss Federal Institute of Technology Global Health Institute, \\ Lausanne CH-1015, Switzerland
}

Received June 11, 2018; Accepted November 8, 2018

DOI: $10.3892 /$ or.2018.6886

\begin{abstract}
The management of advanced ovarian cancer is challenging due to the high frequency of recurrence, often associated with the development of resistance to platinum-based chemotherapy. Molecular analyses revealed the complexity of ovarian cancer with particular emphasis on the immune system, which may contribute to disease progression and response to treatment. Cytokines and chemokines mediate the cross-talk between cancer and immune cells, and therefore, present as potential biomarkers, reflecting the tumor microenvironment. A panel of circulating $\mathrm{C}-\mathrm{C}$ motif chemokine ligand (CCL) and $\mathrm{C}-\mathrm{X}-\mathrm{C}$ motif chemokine ligand (CXCL) chemokines were examined in the serum of 40 high-grade patients with ovarian cancer prior to primary surgery. The level of immune infiltration in tumors was also analyzed. The preoperative levels of chemokines differ between patients. Elevated levels of circulating CXCL4 + CCL20 + CXCL1 combination can discriminate patients with shorter recurrence-free survival and overall survival. The presence of tumor-infiltrating $\mathrm{T}$ lymphocytes was detected in half of the patients. The mRNA expression analysis suggests the presence of antitumoral and immunosuppressive elements in the tumor microenvironment. The combination of circulating CXCL9+CXCL10 can distinguish immune-infiltrated tumors that will lead to shorter recurrence-free survival. The results suggest that preoperative profiling of circulating chemokines in patients with ovarian cancer may provide valuable information regarding tumor recurrence and immune infiltration. The findings demonstrate
\end{abstract}

Correspondence to: Ms. Agata Mlynska, Laboratory of Immunology, National Cancer Institute, 1 Street Santariskiu, Vilnius LT-08660, Lithuania

E-mail: agata.mlynska@gmail.com

Key words: ovarian neoplasms, recurrence, chemokines, biomarkers, immunity that combinations have better prognostic utility than single chemokines, and may serve as patient stratification tools.

\section{Introduction}

The current situation in ovarian cancer (OC) management remains unsatisfactory, as the overall survival has hardly improved in the past decades $(1,2)$. High-grade serous ovarian carcinoma (HGSOC), the most commonly diagnosed histological subtype, nearly always presents as advanced disease, and therefore, is associated with low overall survival (3). The standard treatment for primary $\mathrm{OC}$ is cytoreductive surgery followed by six cycles of adjuvant chemotherapy with carboplatin and paclitaxel. HGSOC tumors are initially responsive to platinum-based chemotherapy; however, the majority of patients eventually experience tumor recurrence (4). Platinum-free interval (PFI), the period from completion of primary chemotherapy to disease recurrence, serves as measure to further subdivide OC patients into the following groups based on their response to platinum: Refractory (PFI <1 month), resistant (PFI 1-6 months), par-sensitive (PFI 6-12 months) and sensitive (PFI >12 months) (5). Although currently this grouping serves as a practical guideline for secondary chemotherapy selection, it has limitations. Multiplex categorization of patients, incorporating the recent discoveries of molecular genetics, is suggested for future patient stratification in clinical trials (6).

Molecular analysis of HGSOC revealed its underlying genomic instability, DNA repair defects and copy number alterations (7). Furthermore, several independent groups were classified as HGSOC based on gene and microRNA expression patterns, with four largely overlapping molecular subtypes: $\mathrm{C} 1 /$ mesenchymal, C2/immunoreactive, C4/differentiated and C5/proliferative $(7,8)$. Later studies emphasized an active role of the stromal tumor microenvironment in the pathogenesis of HGSOC and presented evidence for association between molecular subtypes and survival $(9,10)$, in particular that the immunoreactive subtype-bearing patients, characterized by 
the elevated mRNA expression of chemokines, major histocompatibility complex class I/II, programmed cell death 1 ligand 1 (PD-L1), and interferon regulatory factor 7 , have a better prognosis than the other subtypes (11). These findings emphasize the role of the immune system in OC, previously suggested in the study by Hwang et al (12), which demonstrated that tumor infiltrating lymphocytes (TILs) were correlated with increased overall survival in patients with OC. Recently, an immune score for OC was proposed, linking the compendium of immune marker genes with response to chemotherapy and survival. The infiltration of the majority subpopulations associated adaptive immunity [except regulatory $\mathrm{T}$ cells (Tregs)] was associated with favorable prognosis (13).

The role of the immune system in the development of chemoresistance and recurrence of $\mathrm{OC}$ remains elusive. The primary evidence for association of immune cells and chemosensitivity in $\mathrm{OC}$ was recently reported from in vitro and mouse model studies, which demonstrated the ability of T-cell surface glycoprotein CD8 (CD8) ${ }^{+} \mathrm{T}$ cells to alter the metabolism of cytostatic drugs in fibroblasts (14) or negative regulation of $\mathrm{PD}-\mathrm{L} 1$ on $\mathrm{CD}^{+} \mathrm{T}$ cells $(15)$ to abrogate chemoresistance. However, the dual nature of the immune system is often exploited by tumor cells to create local immune suppression (16) and promote chemoresistance (17), as demonstrated in carcinomas of various origins $(18,19)$. The nature of the cross-talk between cancer and other stromal cells is orchestrated by a cytokine and chemokine network, which can act both locally and systemically (20). The circulating cytokines and chemokines reflect the tumor microenvironment (21) and, therefore, are convenient candidates for the discovery of novel biomarkers as the basis for rational treatment decisions.

Despite frequent recurrence and limited effective treatment options, the selection of reliable prognostic and predictive biomarkers in HGSOC remains limited (22). The present study aimed to evaluate whether the levels of circulating signaling molecules can distinguish patients that are less likely to respond to chemotherapy. The immune profiles of HGSOC tumors were also evaluated to select circulating chemokines that reflect the immune infiltration.

\section{Materials and methods}

Patient cohort. A total of 40 patients with confirmed diagnosis of HGSOC of III-IV International Federation of Gynecology and Obstetrics stage (23) with no prior cancer history or immune disorders were involved in this study. All patients (age range 32-76 years) underwent primary cytoreductive surgery and completed six cycles of adjuvant carboplatin-based chemotherapy between April 2013 and April 2015 at the National Cancer Institute (Vilnius, Lithuania). For each patient, a pre-operative serum sample and surgically removed tumor specimen was collected. Clinical data were obtained from the patients' medical records. Patients were followed up until April 2018 to determine platinum status and recurrence. This study was approved by the Lithuanian Bioethics Committee (approval no. 158200-06-500-147). All patients signed informed consent form.

Sample preparation. Serum was centrifuged at $2,000 \mathrm{xg}$ for $10 \mathrm{~min}$ at $22^{\circ} \mathrm{C}$, aliquoted and stored at $-80^{\circ} \mathrm{C}$ prior to analysis. Tumor tissue was collected during surgery. Fresh tissue was immediately divided into four parts for enzymatic dissociation, protein extraction, RNA extraction and a fresh-frozen backup sample. All samples were processed on the same day. For preparing single cell suspensions, tumor tissue was incubated in digestion solution $[5 \mathrm{mg} / \mathrm{ml}$ collagenase II, $5 \mathrm{mg} / \mathrm{ml}$ collagenase IV and $1 \mathrm{mg} / \mathrm{ml}$ DNase, all Sigma-Aldrich (Merck KGaA, Darmstadt, Germany)] for $30 \mathrm{~min}$ at $37^{\circ} \mathrm{C}$. Following gentle pipetting, the solution was filtered and washed with PBS. For red blood cell lysis, the cell pellet was treated with $5 \mathrm{ml} 1 \mathrm{X}$ BD FACS Lysing solution (BD Biosciences, San Jose, CA, USA) for $10 \mathrm{~min}$ at $22^{\circ} \mathrm{C}$ and washed with PBS. For protein extraction, tumor tissue was homogenized and lysed using T-PER Tissue Protein Extraction Reagent (Thermo Fisher Scientific, Inc., Waltham, MA, USA), supplemented with protease/phosphatase inhibitor cocktail (Thermo Fisher Scientific, Inc.), followed by $15 \mathrm{~min}$ centrifugation at $10,000 \times \mathrm{g}$ at $4^{\circ} \mathrm{C}$ and debris removal. For RNA extraction, tumor tissue was homogenized with TRIzol reagent from the TRIzol Plus RNA Purification kit (Thermo Fisher Scientific, Inc.) and RNA was purified according to the manufacturer's protocol.

Cytokine and chemokine measurement. Serum samples were analyzed using the Proteome Profiler Human Cytokine Array kit (R\&D Systems, Inc., Minneapolis, MN, USA) according to manufacturer's instructions. Membranes with cytokine expression signal were scanned with LI-COR C-DiGit blot scanner (LI-COR Biosciences, Lincoln, NE, USA) and analyzed with ImageJ software (v1.50f; National Institute of Health, Bethesda, MD, USA).

A panel of 13 chemokines [C-C motif chemokine ligand (CCL) 2, -3, -4, -5, -11, -17 and -20; C-X-C motif chemokine ligand (CXCL) 1, -5, -8, -9, -10 and -11) was measured in all patients serum and tumor lysate samples using LEGENDplex Human Proinflammatory Chemokine Panel (BioLegend, Inc., San Diego, CA, USA), a bead-based immunoassay, according to the manufacturer's guidelines. Samples were assayed in duplicates in 96-well plates, collected with BD LSR II flow cytometer (BD Biosciences) and analyzed with LEGENDplex data analysis software (V7.1; BioLegend, Inc.).

Evaluation of gene expression by reverse transcription-quantitative polymerase chain reaction $(R T-q P C R)$. For cDNA synthesis, $500 \mu \mathrm{g}$ RNA from each sample was reverse transcribed using Maxima First Strand cDNA Synthesis kit (Thermo Fisher Scientific, Inc.) according to the manufacturer's protocol. qPCR was performed in duplicate in Eco Real-Time thermocycler (Illumina, Inc., San Diego, CA, USA). The reaction volume of $10 \mu \mathrm{l}$ contained $5 \mu \mathrm{l}$ Maxima SYBR-Green qPCR Master Mix 2X (Thermo Fisher Scientific, Inc.), $2.5 \mu 10.8 \mu \mathrm{mol} / 1$ sequence-specific forward and reverse primers mix, $1 \mu \mathrm{l}$ cDNA reaction product, and $1.5 \mu \mathrm{l}$ nuclease-free water. The reaction was started by $5 \mathrm{~min}$ at $95^{\circ} \mathrm{C}$ and continued with 40 cycles of $10 \mathrm{sec}$ denaturing at $95^{\circ} \mathrm{C}$ and $30 \mathrm{sec}$ of annealing/extension at $60^{\circ} \mathrm{C}$. Primer sequences are listed Table I. The expression level of selected genes was evaluated with EcoStudy software (Illumina, Inc.), using GAPDH and ribosomal protein L13 as the reference genes. The analysis was performed using $\Delta \Delta \mathrm{Cq}$ relative quantitation method (24) with Pfaffl correction for PCR 
Table I. Primer sequences.

\begin{tabular}{llll}
\hline Gene ID & Protein alias & \multicolumn{1}{c}{ Forward primer (5'-3' sequence) } & Reverse primer (5'-3' sequence) \\
\hline$C D 163$ & CD163 & CGAGTTAACGCCAGTAAGG & GAACATGTCACGCCAGC \\
$C D 274$ & PD-L1 & TATGGTGGTGCCGACTACAA & TGGCTCCCAGAATTACCAAG \\
$C D 4$ & CD 4 & TGCCTCAGTATGCTGGCTCT & GAGACCTTTGCCTCCTTGTC \\
$C D 68$ & CD68 & TGGGGCAGAGCTTCAGTTG & TGGGGCAGGAGAAACTTTGC \\
$C D 8 A$ & CD8 & ACTTGTGGGGTCCTTCTCCT & GTCTCCCGATTTACCACAG \\
$C T L A 4$ & CTLA-4 & TGCAGCAGTTAGTTCGGGGTTGTT & CTGGCTCTGTTGGGGCATTTC \\
$C X C L 10$ & CXCL10 & AAGGATGGACCACACAGAGG & ACCCTTGGAAGATGGGAAAG \\
$C X C L 11$ & CXCL11 & ATGAGTGTGAAGGGCATGGC & TCACTGCTTTACCCCAGG \\
$E O M E S$ & EOMES & AGCTCTCCAAGGAGAAAGTG & GCCTTCGCTTACAAGCACTG \\
$F C G R 2 A$ & CD32 & TTTGAGATGAGTAATCCCAGCCA & TCAGGCCCAGTCTCCATTTA \\
$F O X P 3$ & FOXP3 & GAACGCCATCCGCCACAACCTGA & CCCTGCCCCCACCACCTCTGC \\
$G A P D H$ & GAPDH & AATCCCATCACCATCTTCCA & TGGACTCCACGACGTACTCA \\
$G Z M B$ & Granzyme B & GAAACGCTACTAACTACAGG & CCACTCAGCTAAGAGGT \\
$I D O 1$ & IDO & GGCAAAGGTCATGGAGATGT & CAGGACGTCAAAGCACTGAA \\
$I F N G$ & IFN $\gamma$ & TGGAAAGAGGAGAGTGACAGAA & TCTTTTGATGCTCTGGTCAT \\
$I L 10$ & IL-10 & GACTTTAAGGGTTACCTGGGTTG & TCACATGCGCCTTGATGTCTG \\
$P D C D 1$ & PD-1 & ACCCTGGTCATTCACTTGGG & CATTTCTCCCTCTGACACTG \\
$P S M B 9$ & PSMB9 & GCACCAACCGGGGACTTAC & CACTCGGGAATCAGAACCCAT \\
$R L P 13$ & RLP13 & GAGGTATGCTGCCCCACAA & GTGGGATGCCGTCAAACA \\
$T A P 1$ & TAP1 & TGCCCCGCATATTCTCCCT & CACCTGCGTTTTCGCTCTTG \\
$T A P 2$ & TAP2 & TGGACGCGGCTTTACTGTG & GCAGCCCTCTTAGCTTTAGCA \\
$T I G I T$ & TIGIT & TCTGCATCTATCACACCTACCC & CCACCACGATGACTGCTGT \\
\hline
\end{tabular}

efficiency (25). Relative gene expression was z-transformed, visualized and clustered using K-means clustering by Euclidean distance for selection of non-inflamed (z-score negative) and inflamed patient (z-score positive) clusters with Morpheus software (Broad Institute, Cambridge, MA, USA).

Flow cytometry. Single tumor cell suspension was stained for $20 \mathrm{~min}$ at $4^{\circ} \mathrm{C}$ with pre-titrated amounts of monoclonal mouse anti-human antibodiesprotein tyrosine phosphatase, receptor type C (CD45)-V450 (cat. no. 560367; BD Biosciences) at 1:20 dilution and T-cell surface glycoprotein CD3 (CD3)-allophycocyanin (cat. no. 300312; BioLegend, Inc.) at 1:50 dilution. Cells were washed, stained with $5 \mu \mathrm{g} / \mathrm{ml}$ viability dye 7-aminoactinomycin D (BD Biosciences) for $5 \mathrm{~min}$ at $22^{\circ} \mathrm{C}$, collected with BD LSR II flow cytometer (BD Biosciences), and analyzed using BD FACSDiva software (v6.2; BD Biosciences).

Histological assessment of tumor tissue. Tumor tissue was fixed in $4 \%$ paraformaldehyde (Sigma-Aldrich; Merck KGaA) for $24 \mathrm{~h}$ at $22^{\circ} \mathrm{C}$. Tissue sections from paraffin-embedded blocks ( $4 \mu \mathrm{m}$-thick) were stained with Mayer's hematoxylin solution for $40 \mathrm{sec}$ and $1 \%$ eosin for $30 \mathrm{sec}$ (H\&E; all SigmaAldrich; Merck KGaA) at $22^{\circ} \mathrm{C}$. Tumor type and grade were assessed $(26,27)$. Qualitative evaluations for the presence of either intraepithelial or stromal T lymphocytes within tumor tissue were conducted by the pathologist.

Statistical analysis. Data were analyzed and visualized using GraphPad Prism 7 (GraphPad Software, Inc., La Jolla,
CA, USA) statistical software. The associations between systemic and local chemokine expression and clinical characteristics were evaluated by the Spearman rank correlation coefficient. Differences in chemokine levels between patients were determined with a Mann-Whitney U test. Differences in patient characteristics between groups were identified by Kruskal-Wallis test. Receiver operator characteristic (ROC) curves were created to determine the predictive performance of the cytokines and their combinations. The area under curve (AUC), sensitivity and specificity were calculated from ROC curves. Performance metrics and clinical utility were calculated and converted into qualitative grades: Excellent utility, $\geq 0.81$; good, $\geq 0.64$; fair, $\geq 0.49$; and poor, $<0.49$, as suggested previously (28). To combine chemokines, logistic regression was applied. The best combination in each setting was selected based on the AUC and sensitivity. For combinations with equally good characteristics, a classifier with the least number of elements was chosen. The Kaplan-Meier survival curves and hazard ratios were analyzed with a log-rank test. Cohort clinicopathological features were compared with the $\chi^{2}$ test. $\mathrm{P}<0.05$ was considered to indicate a statistically significant difference. Given the exploratory nature of this study, P-values were not adjusted for multiple testing.

\section{Results}

Patient characteristics. All patients involved in this study $(n=40)$ were diagnosed with stage III $(90 \%)$ or IV $(10 \%)$ HGSOC. Following the complete resection of tumor foci, all patients completed six cycles of adjuvant chemotherapy with 
Table II. Overview of patient characteristics $(n=40)$.

\begin{tabular}{|c|c|c|c|c|c|}
\hline Feature & $\begin{array}{c}\text { Pt-resistant } \\
\text { (PFI }<6 \text { months) }\end{array}$ & $\begin{array}{l}\text { Pt-par-sensitive } \\
\text { (PFI 6-12 months) }\end{array}$ & $\begin{array}{l}\text { Pt-sensitive } \\
\text { (recurrent PFI >12 months) }\end{array}$ & $\begin{array}{c}\text { Pt-sensitive } \\
\text { (non-recurrent) }\end{array}$ & P-value \\
\hline $\mathrm{N}$ & 15 & 7 & 7 & 11 & \\
\hline Age & & & & & 0.335 \\
\hline Median (years) & 62 & 69 & 60 & 67 & \\
\hline Range & $53-76$ & $53-75$ & $32-72$ & $32-74$ & \\
\hline Stage & & & & & 0.639 \\
\hline III & $13(87 \%)$ & $6(86 \%)$ & $6(86 \%)$ & $11(100 \%)$ & \\
\hline IV & $2(13 \%)$ & $1(14 \%)$ & $1(14 \%)$ & $0(0 \%)$ & \\
\hline RFS (median months) & 4.1 & 9.7 & 21.0 & NR & $<0.0001$ \\
\hline OS (median months) & 27.5 & 21.0 & NR & NR & 0.0024 \\
\hline
\end{tabular}

Differences between groups were identified by Kruskal-Wallis test. Pt, platinum; PFI platinum-free interval; RFS, recurrence-free survival; OS, overall survival; NR, not reached.
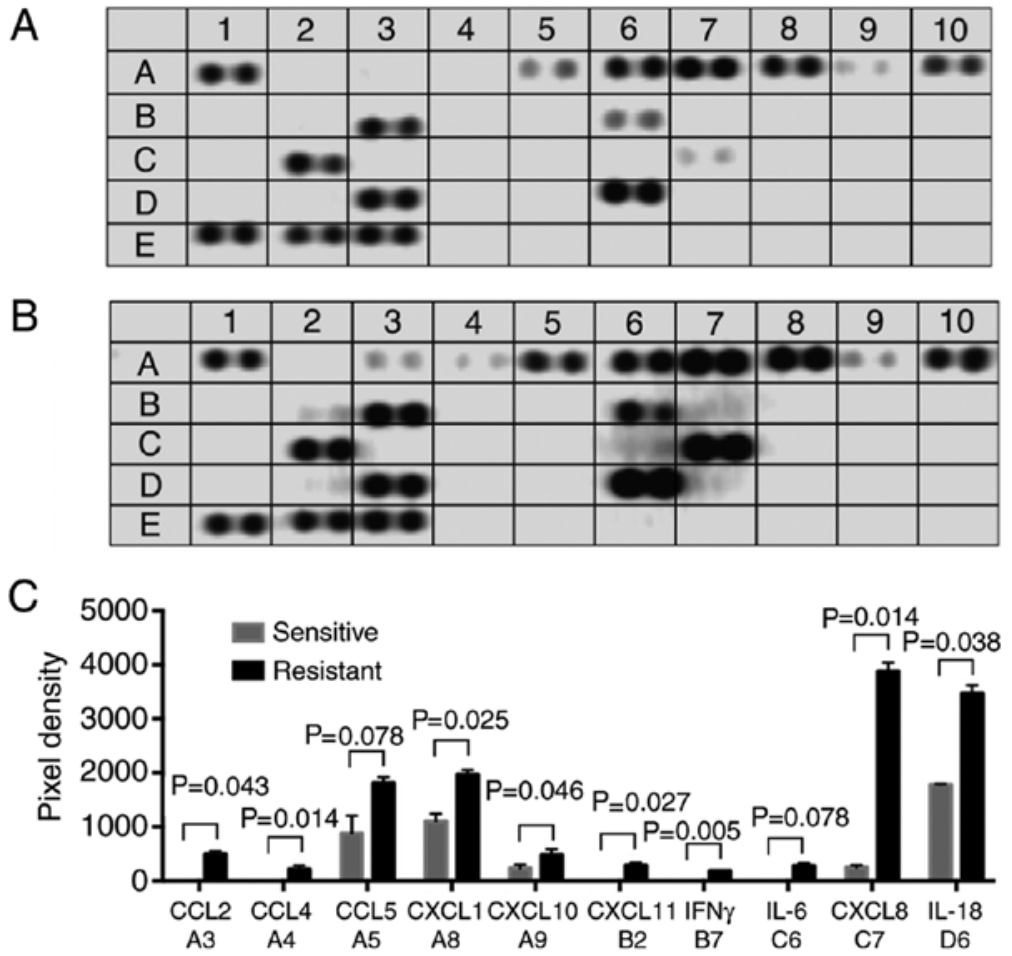

Figure 1. Serum cytokine levels in patients with ovarian cancer. The representative cytokine array membrane scans of (A) sensitive and (B) resistant patients ( $n=4$ for each group) are presented. (C) Semi-quantitative results of differentially expressed cytokines, presented as mean with standard deviation and compared using Mann-Whitney U test. CCL, C-C motif chemokine ligand; CXCL, C-X-C motif chemokine ligand.

carboplatin and paclitaxel. The median follow up time was 46 months. The patients were regularly tested for the blood CA125 level. Recurrence was confirmed by radiological imaging. Recurrence of primary disease occurred in $73 \%$ of patients $(n=29)$ during the follow-up period, with median recurrence-free survival (RFS) of 11.1 months. Recurrent patients were classified as platinum-resistant (PFI $<6$ months), par-sensitive (PFI 6-12 months) or sensitive (recurrent PFI $>12$ months or non-recurrent) based on the duration of PFI. An overview of patient clinical characteristics is presented in Table II.
Inflammatory serum protein profiling reveals differential chemokine expression among HGSOC patients. It was hypothesized that profiling of multiple inflammatory markers could help unveil the role of inflammation in ovarian cancer progression. The relative level of 36 different cytokines, chemokines and acute phase proteins was analyzed in the serum of platinum-resistant (Fig. 1A) and non-recurrent platinum-sensitive patients (Fig. 1B; n=4 for each group). Out of 18 proteins detected, 10 proteins were to be more abundant in sera of platinum-resistant patients compared with platinum-sensitive patients (Fig. 1C). The majority of the proteins were members of the $\mathrm{CC}$ and $\mathrm{CXC}$ chemokine families. 
Table III. Correlations between circulating preoperative serum chemokine concentrations and their gene and protein expression level in tumor, tumor infiltration with immune cells and patient survival.

\begin{tabular}{lccccrr}
\hline $\begin{array}{l}\text { Serum } \\
\text { chemokine }\end{array}$ & $\begin{array}{c}\text { Relative gene } \\
\text { expression of respective } \\
\text { chemokine in tumor }\end{array}$ & $\begin{array}{c}\text { Respective chemokine } \\
\text { concentration in } \\
\text { tumor lysate }\end{array}$ & $\begin{array}{c}\text { Total immune } \\
\text { tumor infiltration } \\
\text { (CD45) }\end{array}$ & $\begin{array}{c}\text { Immune infiltration } \\
\text { with T lymphocytes } \\
\left(\text { CD45 }^{+} / \text {CD3 }^{+}\right)\end{array}$ & RFS & OS \\
\hline CCL2 & 0.206 & 0.196 & 0.302 & $\mathbf{0 . 3 2 7}$ & -0.220 & -0.115 \\
CCL3 & $\mathbf{0 . 6 5 8}$ & 0.202 & 0.200 & 0.214 & $\mathbf{- 0 . 3 8 4}$ & 0.118 \\
CCL4 & 0.175 & 0.245 & 0.095 & 0.234 & -0.210 & -0.162 \\
CCL5 & 0.194 & 0.175 & -0.096 & -0.077 & -0.120 & -0.115 \\
CCL11 & 0.235 & 0.153 & 0.309 & 0.120 & -0.256 & 0.027 \\
CCL17 & 0.246 & 0.261 & -0.034 & -0.121 & 0.024 & 0.060 \\
CCL20 & $\mathbf{0 . 5 9 6}$ & $\mathbf{0 . 3 9 1}$ & 0.029 & 0.008 & $\mathbf{- 0 . 3 2 0}$ & -0.154 \\
CXCL1 & $\mathbf{0 . 3 9 0}$ & $\mathbf{0 . 4 1 0}$ & 0.118 & 0.017 & -0.106 & -0.022 \\
CXCL5 & 0.296 & 0.234 & -0.002 & -0.048 & -0.170 & -0.264 \\
CXCL8 & 0.196 & 0.105 & 0.151 & 0.051 & -0.129 & 0.096 \\
CXCL9 & $\mathbf{0 . 4 2 3}$ & 0.257 & $\mathbf{0 . 3 7 8}$ & $\mathbf{0 . 4 5 8}$ & $\mathbf{- 0 . 4 5 5}$ & -0.295 \\
CXCL10 & $\mathbf{0 . 6 0 7}$ & $\mathbf{0 . 5 1 1}$ & $\mathbf{0 . 4 7 5}$ & $\mathbf{0 . 3 9 3}$ & $\mathbf{- 0 . 3 7 9}$ & 0.067 \\
CXCL11 & $\mathbf{0 . 3 3 5}$ & 0.119 & 0.179 & 0.238 & -0.194 & -0.172 \\
\hline
\end{tabular}

Correlations evaluated by Spearman rank correlation. Data in bold show significant differences with $\mathrm{P}<0.05$. CD45, receptor-type tyrosine-protein phosphatase C; CD3, T-cell surface glycoprotein CD3; RFS, recurrence-free survival; OS, overall survival; CCL, C-C motif chemokine ligand; CXCL, C-X-C motif chemokine ligand.

Therefore, the panel of interest was expanded and the concentration of thirteen selected $\mathrm{CC}$ and $\mathrm{CXC}$ family chemokines was quantified (CCL2, $-3,-4,-5,-11,-17$ and -20 , CXCL1, $-5,-8,-9,-10$ and -11 ) in patient serum samples. To examine how the serum chemokine levels reflect the tumor microenvironment and disease course, the correlation with intratumoral chemokine expression, immune cell infiltration, RFS and overall survival (OS) were assessed (Table III). Although all serum chemokines were positively associated with gene and protein expression in tumors, their correlation coefficients varied in magnitude. Among serum chemokines, CXCL9 and CXCL10 levels were positively correlated with the intratumoral infiltration of $\mathrm{CD}^{+} 5^{+}$cells (total leukocytes) and $\mathrm{CD} 45^{+} / \mathrm{CD}^{+}$cells (total TILs), as measured by flow cytometry. These two chemokines, along with CCL3 and CCL20, were significantly negatively associated with RFS. Serum chemokines did not have any significant correlations with OS.

Combination of circulating CCL4 +CXCL1 +CCL2O predicts recurrence-prone tumors. Due to the association of preoperative circulating chemokine levels with RFS, the patients were subsequently grouped based on their recurrence status and the serum chemokine expression patterns were analyzed. Significantly higher levels of CCL3, CCL4, CCL20 and CXCL1 were present in recurrent patient serum compared with non-recurrent patients (Table IV). To evaluate the predictive value of each chemokine, ROC curve analysis was performed to calculate the cut-off, area under curve (AUC) and sensitivity at clinically relevant specificity. Among single chemokines, CCL4 had the best sensitivity (62\%) and fair positive clinical utility (CUI) at a cut-off of $20 \mathrm{pg} / \mathrm{ml}$. Subsequently, the performance of single chemokines was compared with their multiple combinations in order to select the best biomarkers that may potentially identify the patients in a higher recurrence risk group. All possible double, triple and quadruple combinations were assessed, and then the combination with the highest AUC and least number of elements was selected. In the recurrent cancer setting, combining CCL4, CXCL1 and CCL20 into a single classifier resulted in $81 \%$ sensitivity. The combination of CCL4+CXCL1 + CCL20 also had good positive and fair negative clinical utility. ROC curves for CCL4 and CCL4+CXCL1 + CCL20 classifier highlight the advantage of the chemokine combination (Fig. 2A). The combination of CCL4 + CXCL1 + CCL20 could also predict RFS and OS (Fig. 2B and C). The individuals with elevated serum levels of these three markers had greater chances of disease recurrence $(\mathrm{HR}=4.08)$ and worse survival prognosis $(\mathrm{HR}=5.10)$.

Subsequently, it was sought to determine whether preoperative circulating chemokine levels were able to predict the PFI. There was no difference in serum chemokine concentrations in patients with PFI $<6$ months compared with PFI $>6$ months. However, CCL2, CXCL9, CXCL10 and CCL11 concentrations were higher in the serum of patients with PFI $<12$ months compared with PFI $>12$ months (Table IV). The sensitivity of single markers at $94 \%$ specificity varied between 26 and $40 \%$, resulting in the unsatisfactory clinical utility of these markers. Combining CXCL9+ CXCL10 or CXCL9 + CXCL10 + CCL11 resulted in increased sensitivity (44 and 50\%, respectively). However, the AUC values of either single chemokines or their combinations did not surpass 0.750 (Fig. 2D). Nevertheless, patients with higher preoperative levels of CXCL9+CXCL10+CCL11 were more likely experience recurrence within 12 months (HR=3.05; Fig. 2E). No significant association with OS was observed (Fig. 2F). 


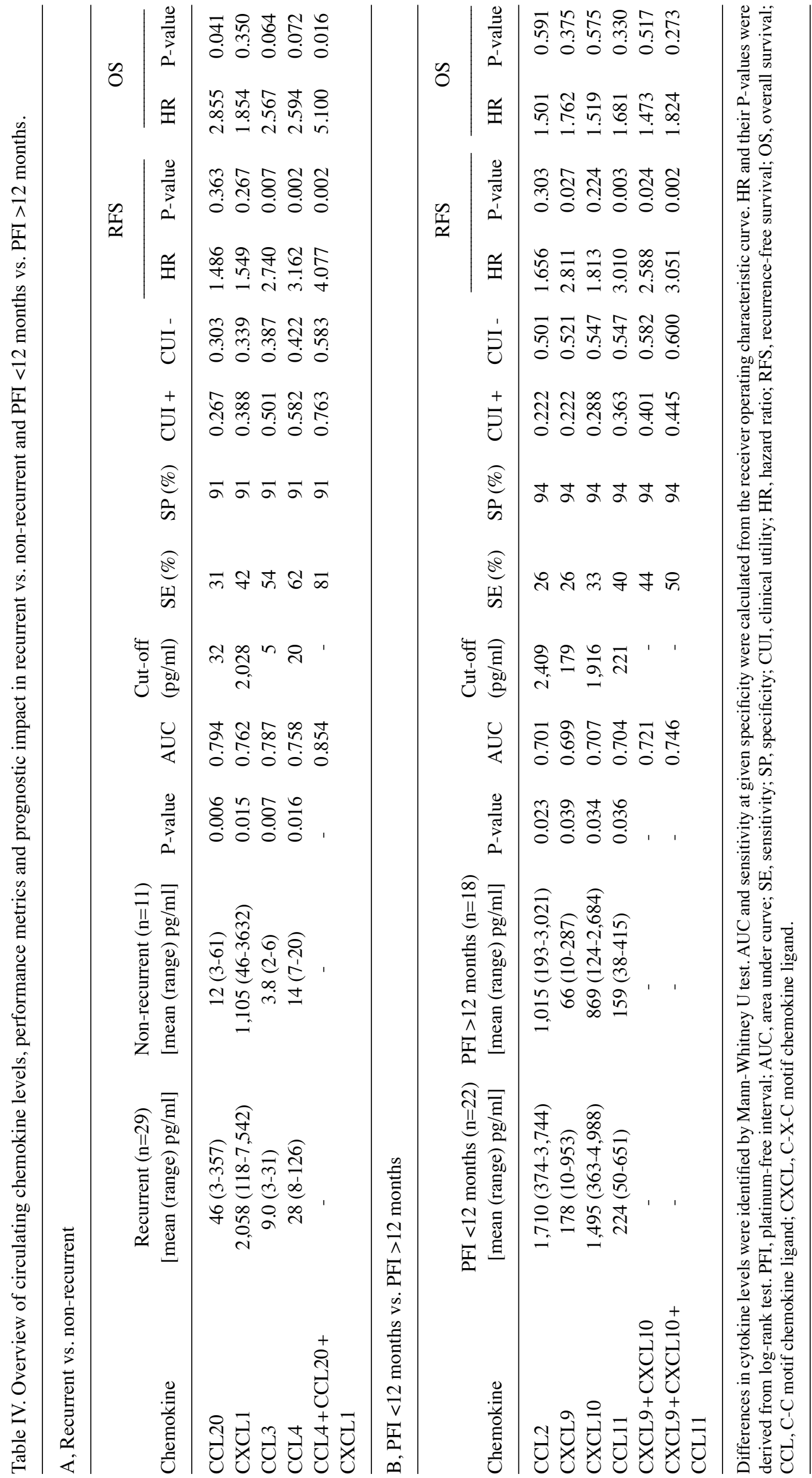


A

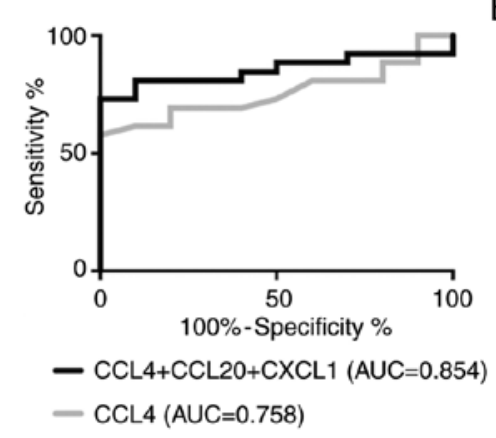

B

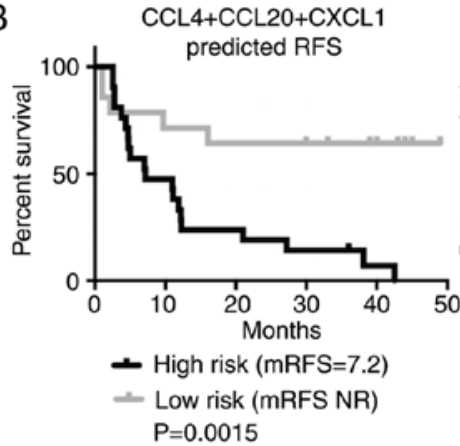

Actual RFS

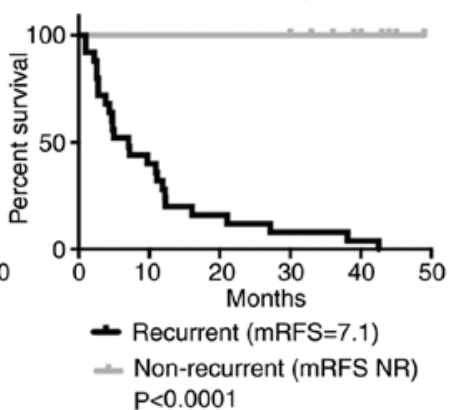

C
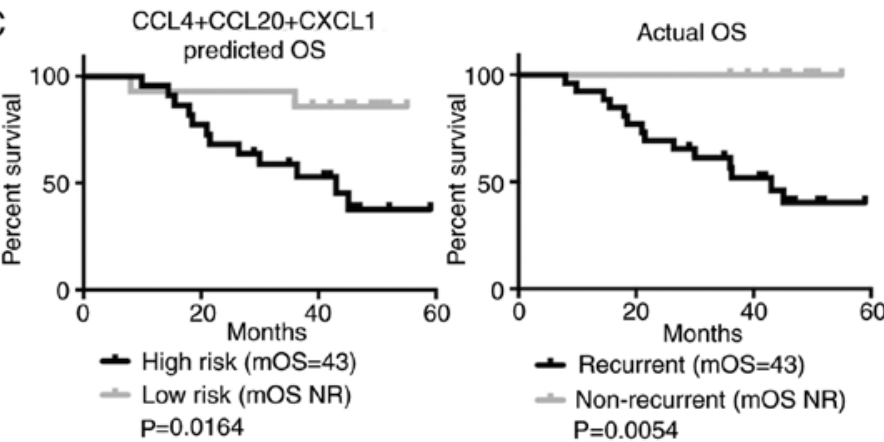

D

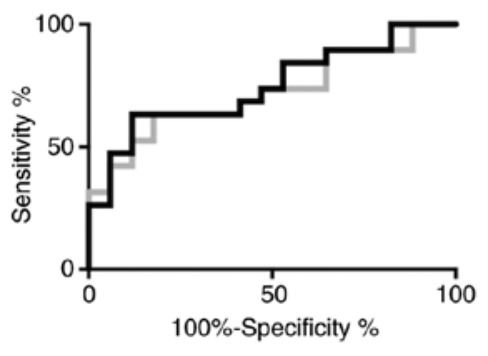

- CXCL9+CXCL10+CCL11 (AUC=0.746)

- CXCL9+CXCL10 (AUC=0.721)

E

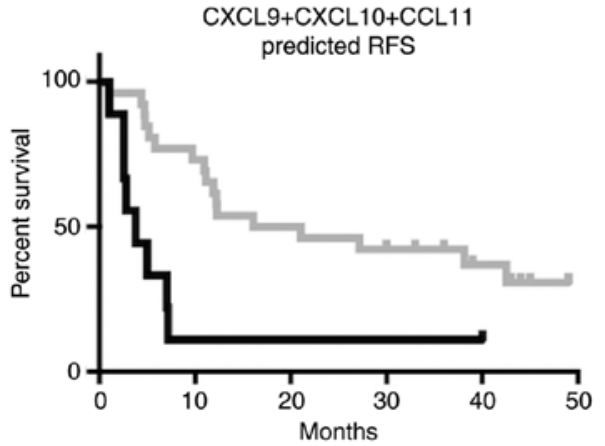

ـ High risk (mRFS=3.8)

ㄴ. Low risk (mRFS=18.6) $\mathrm{P}=0.0019$

$\mathrm{F}$

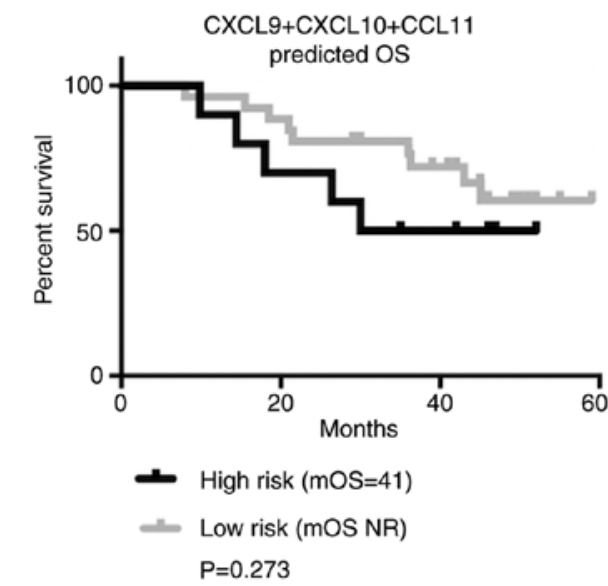

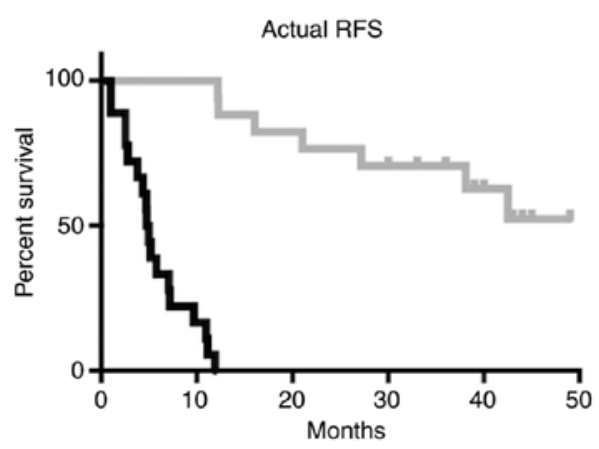

ـ $\mathrm{PFI}<12$ months (mRFS=2.8)

- $\mathrm{PFI}>12$ months (mRFS NR) $\mathrm{P}<0.0001$

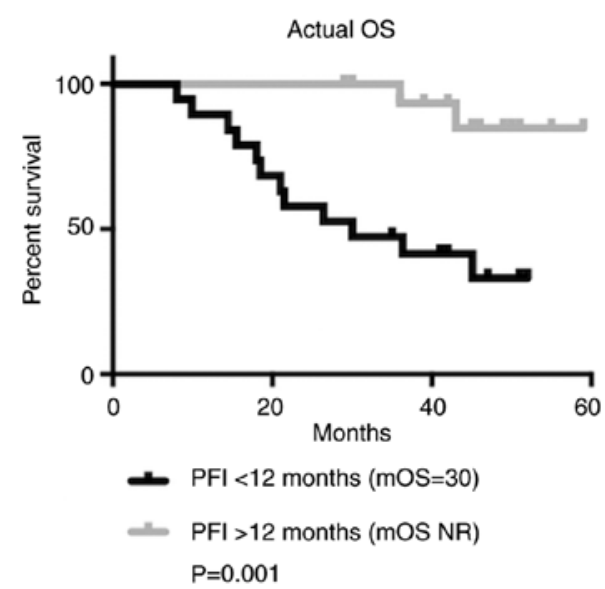

Figure 2. Performance and prognostic impact of CCL4+CCL20+CXCL1 (upper panels) and CXCL9+CXCL10+CCL11 (lower panels). (A) ROC curves of CXCL4 + CCL20 + CXCL1 and CCL4 alone in detecting recurrence-prone patients. (B) RFS and (C) OS estimates for patients with high risk or low risk of recurrence as predicted with CCL4+CCL20+CXCL1, compared to actual survival in recurrent vs. non-recurrent patients. (D) ROC curves of CXCL9+CXCL10 and CXCL9+CXCL10+CCL11 in detecting patients with PFI $<12$ months. (E) RFS and (F) OS survival estimates for patients with high risk or low risk of early ( $<12$ months) recurrence as predicted with CXCL9+CXCL10 + CCL11, compared to actual survival in patients with PFI $<12$ months vs. PFI $>12$ months. mRFS and mOS were calculated from Kaplan Meier survival curves. Survival curves were compared with log-rank test. ROC, receiver operating characteristic; CCL, C-C motif chemokine ligand; CXCL, C-X-C motif chemokine ligand; AUC, area under curve; RFS, recurrence-free survival; mRFS, median RFS; NR, not reached; OS, overall survival; mOS, median OS; PFI, platinum-free interval. 
A

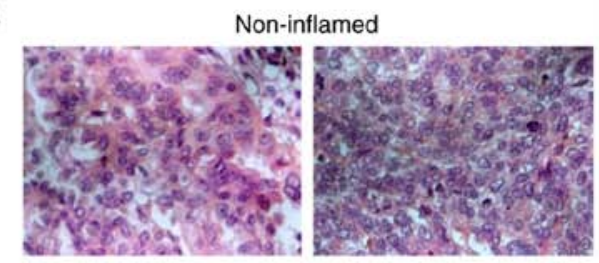

Inflamed

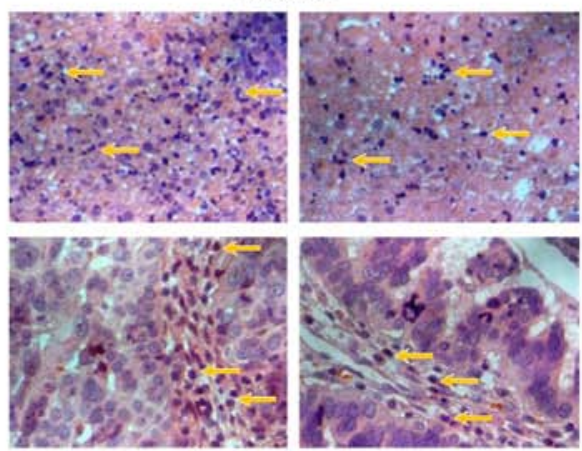

C

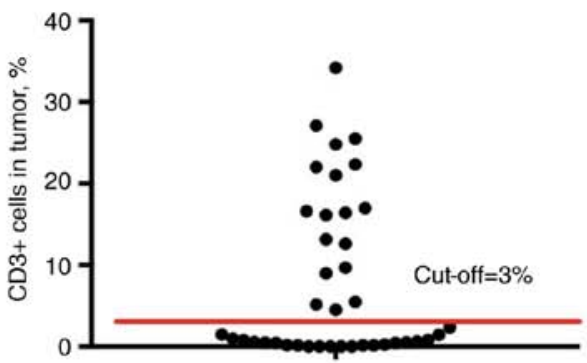

D

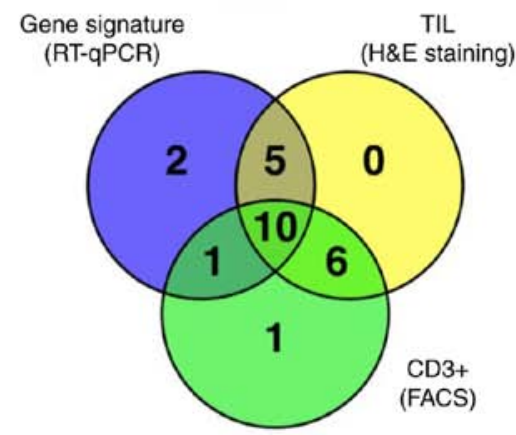

B

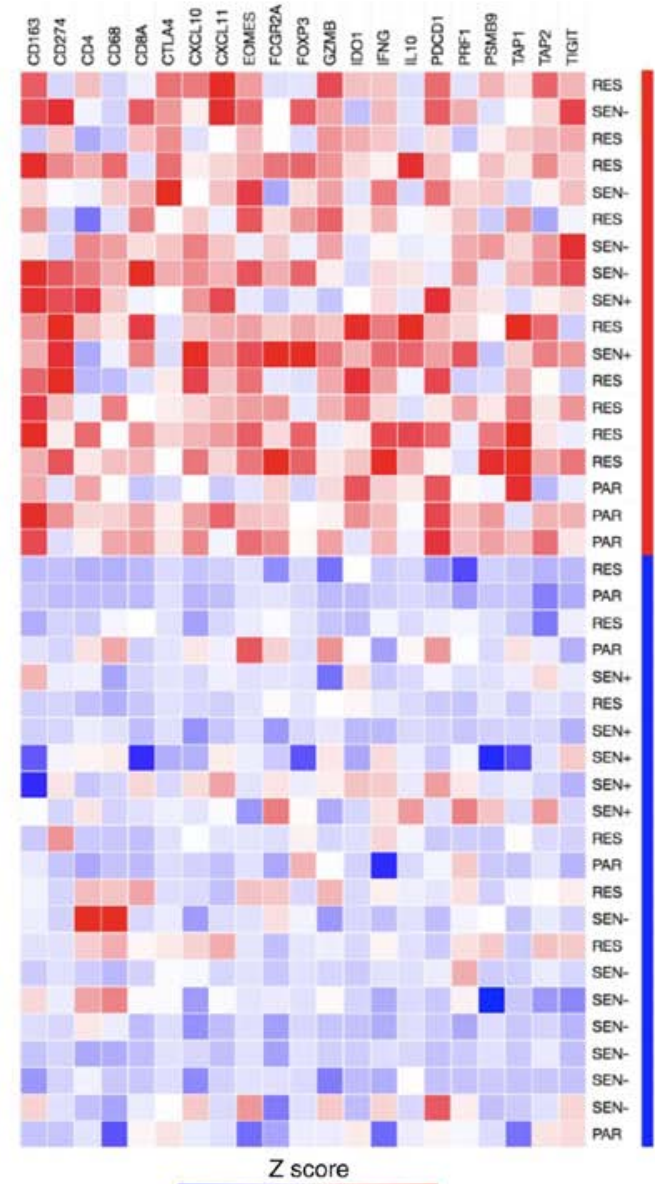

$- 4 \longdiv { 4 }$

E

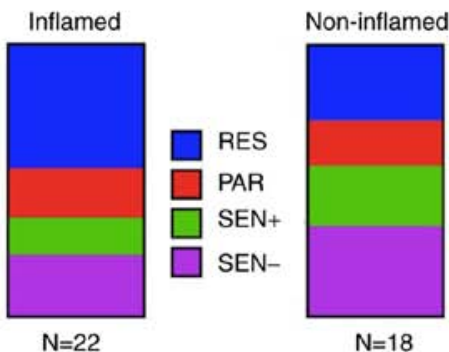

Figure 3. Selection of inflamed tumors. Patients were grouped based on three independent evaluations. (A) Tumors were classified as inflamed if intraepithelial or stromal T lymphocytes (indicated by arrows) were detected in H\&E-stained tissues. (B) Z-transformed tumoral mRNA expression levels of immune response-associated genes, as measured with RT-qPCR, served as a basis for unsupervised clustering of patients into non-inflamed (z score below 0) and inflamed (z score above 0 ) groups (C) Level of $\mathrm{CD}^{+}$cells in tumor samples was evaluated using flow cytometry and a cut-off of 3\% was applied to distinguish immune-infiltrated tumors. (D) Patients were assigned to the inflamed group if positive for at least two factors. (E) Inflamed and non-inflamed groups had a different proportional distribution of patients, based on their platinum sensitivity and recurrence status. CD3, T-cell surface glycoprotein CD3; RT-qPCR, reverse transcription-quantitative polymerase chain reaction; TIL, tumor infiltrating lymphocyte; H\&E, hematoxylin and eosin; RES, platinum-resistant; PAR, partially platinum-sensitive; SEN+, recurrent platinum-sensitive; SEN, non-recurrent platinum-sensitive.

Immune-infiltrated tumors express more intratumoral chemokines. The patient cohort was divided into inflamed and non-inflamed tumor groups, based on three parameters: TILs in tumor sections, immune response-related gene expression in tumor tissue and $\mathrm{CD}^{+}$positive cell count in the tumor (Fig. 3). H\&E-stained tumor tissue sections were evaluated by a pathologist for the presence or absence of both intraepithelial and stromal TILs (Fig. 3A), resulting in 21 TIL-positive and 19 TIL-negative samples. Immune response-associated gene expression was measured with RT-qPCR, and clustered into high-expression (18 samples) and low expression (22 samples) clusters (Fig. 3B). The percentage of $\mathrm{CD}^{+}$cells in tumors was evaluated with flow cytometry analysis of freshly digested tumor tissue samples. Gating for live cells and $\mathrm{CD} 45^{+}$was applied. A cut-off of $3 \% \mathrm{CD}^{+}$cells was applied to divide the study population into 18 samples with high and 22 samples with low immune infiltration in the tumor (Fig. 3C).

The distribution and overlap of samples, identified as TIL-positive, $>3 \% \mathrm{CD}^{+}$or high expression of immune-associated genes, is presented as a Venn diagram in Fig. 3D. Patients were assigned to the inflamed group if they were positive for at least two factors. Out of 25 samples, characterized by at 


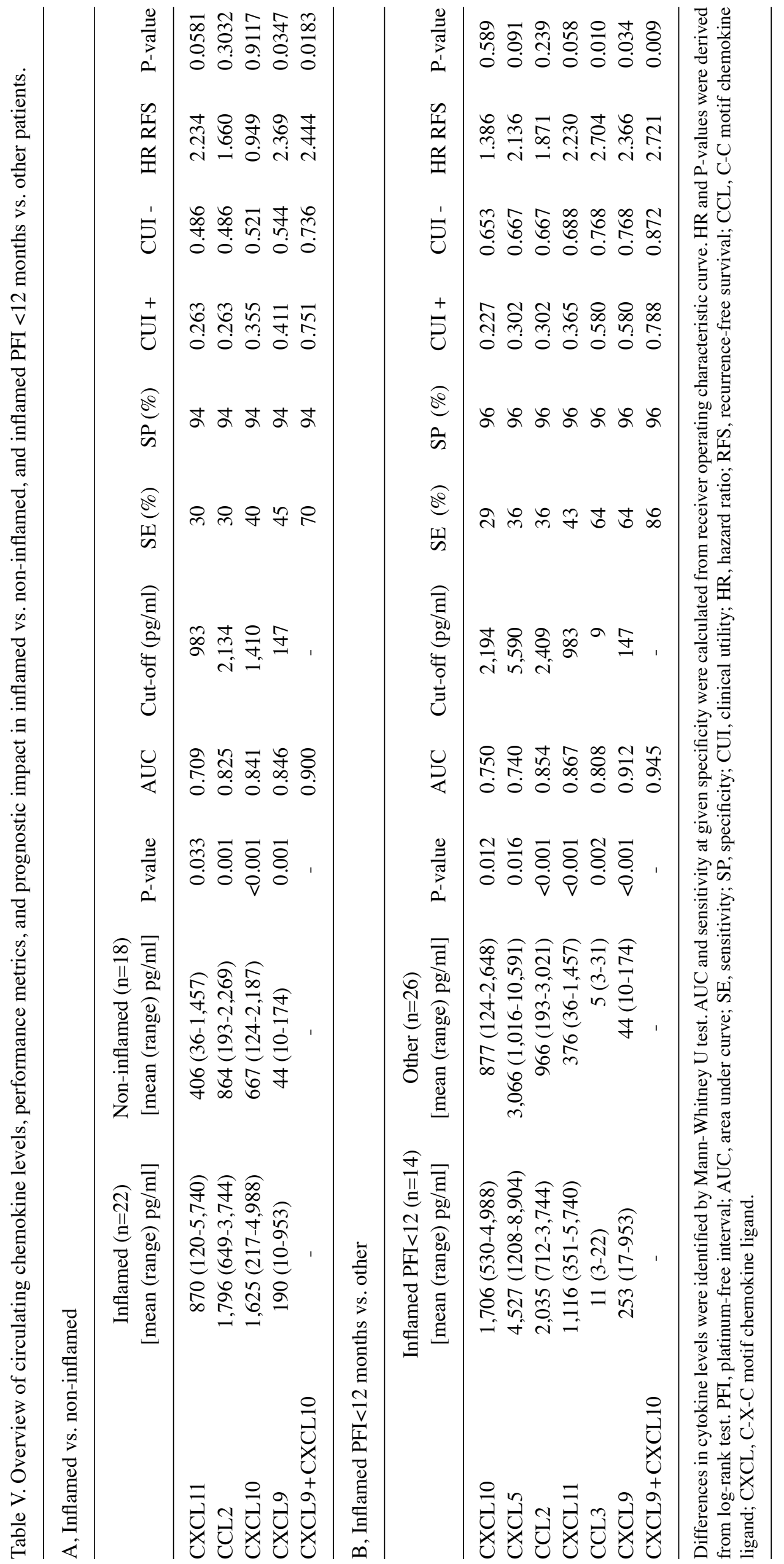



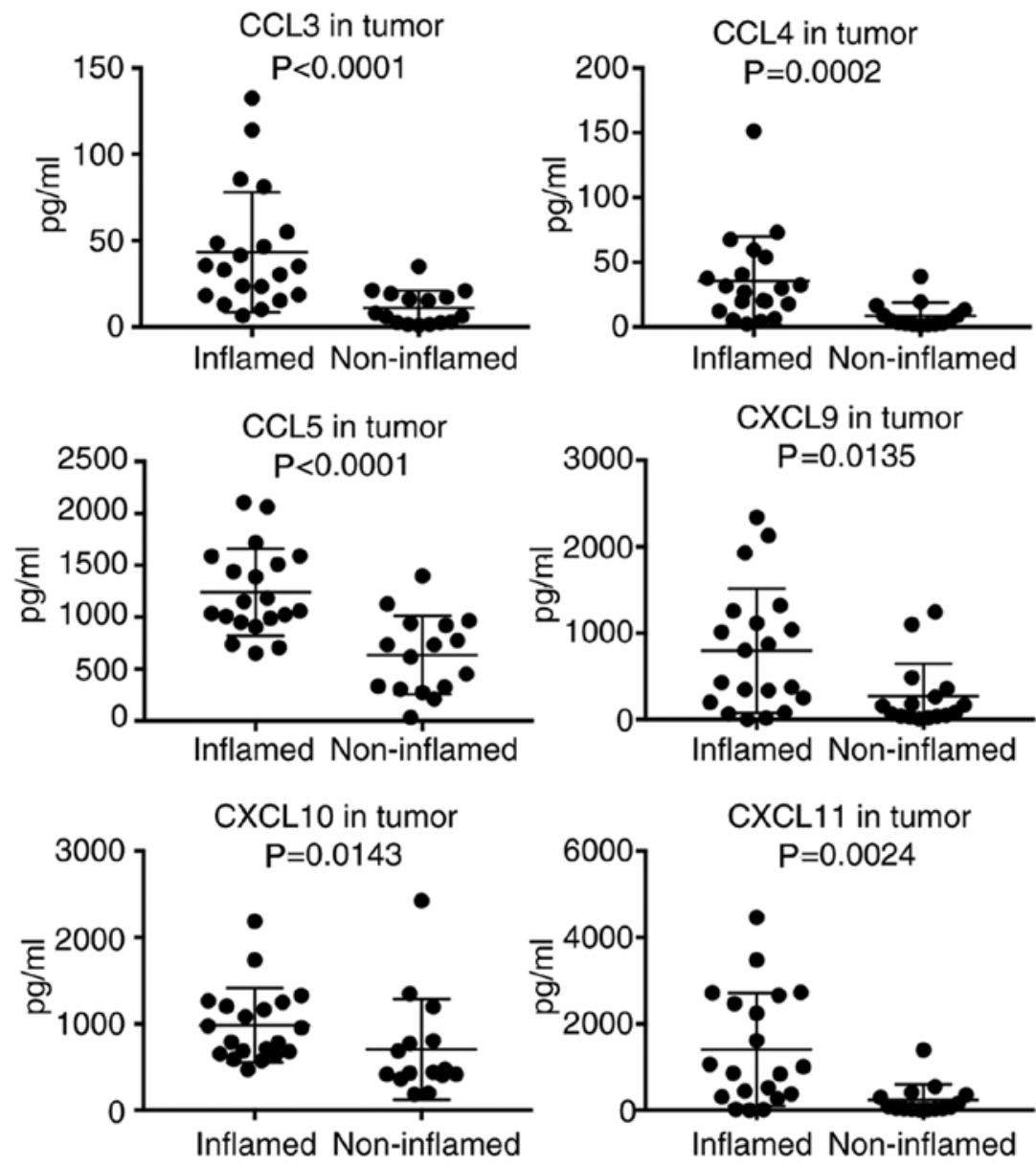

Figure 4. Inflamed tumors have increased levels of intratumoral chemokines. The concentration of chemokines was normalized to total protein concentration in tumor lysate. Scatter plots include mean \pm standard deviation. Differences in cytokine levels were identified by Mann-Whitney U test. CCL, C-C motif chemokine ligand; CXCL, C-X-C motif chemokine ligand.

least one positive inflammation-related factor, only $12 \%$ did not overlap with other factors, and thus were assigned to the non-inflamed group, together with the rest of the samples, which did not qualify for the inflamed group. Finally, 22 patients were allocated to the inflamed and 18 in the non-inflamed group. The proportional distribution of patients based on their platinum-resistance level and recurrence is presented in Fig. 3E.

This classification allowed identification of other significant differences between the groups, including the increased level of CCL3, CCL4, CCL5, CXCL9, CXCL10 and CXCL11 (Fig. 4) in tumors from the inflamed group compared with the non-inflamed group.

Combination of circulating CXCL9+CXCL10 detects inflamed tumors. Following grouping of the patients based on their tumor inflammation level, differences in serum level of CXCL11, CCL2, CXCL10 and CXCL9 were observed. The levels of these chemokines were significantly higher in the serum of patients in the inflamed group compared with the non-inflamed group (Table V). At 94\% specificity, the sensitivity of single chemokines varied between 30 and $45 \%$. Taken alone, these cytokines exhibited poor positive and fair negative clinical utility, despite good AUC values $(>0.8)$. Following screening of all possible multiple chemokine combinations, the CXCL9 + CXCL10 classifier performed best, with $70 \%$ sensitivity and $94 \%$ specificity it resulted in good positive and good negative clinical utility for discrimination of patients with inflamed tumors. CXCL9 + CXCL10 had improved AUC (0.900) compared with CXCL9 alone (0.846; Fig. 5A). Although the actual RFS curves of patients identified as inflamed or non-inflamed did not differ significantly $(\mathrm{p}=0.4102)$, patients with elevated serum levels of CXCL9 + CXCL10 had worse RFS (HR=2.44; Fig. 5B).

Circulating CXCL9 and CXCL10 were the two cytokines able to distinguish between inflamed and non-inflamed tumors, and patients with PFI $<12$ months and PFI $>12$ months, which encouraged assessment of whether circulating chemokines are able to detect immune-infiltrated tumors, which are more likely to recur within 12 months after completion of chemotherapy. For this, inflamed tumors group was narrowed into inflamed with PFI $<12$ months $(n=14)$. The patients in the inflamed PFI $<12$ months group exhibited higher serum levels of CXCL10, CXCL5, CCL2, CXCL11, CCL3 and CXCL9 compared with the remaining patients, with the sensitivity of single chemokines varying from 29-64 at 96\% specificity (Table V). CCL3 and CXCL9 alone had positive and good negative clinical utility. However, the AUC of CXCL9 was the best out of all single markers analyzed. Despite numerous potential multiple chemokine combinations, CXCL9+CXCL10 
A

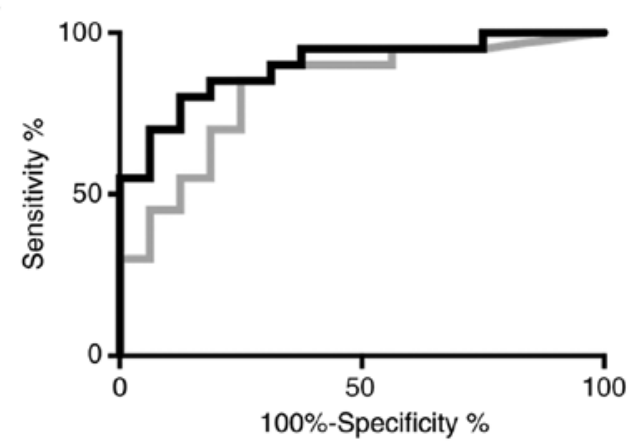

- CXCL9+CXCL10 (AUC=0.900)

- CXCL9 (AUC=0.846)

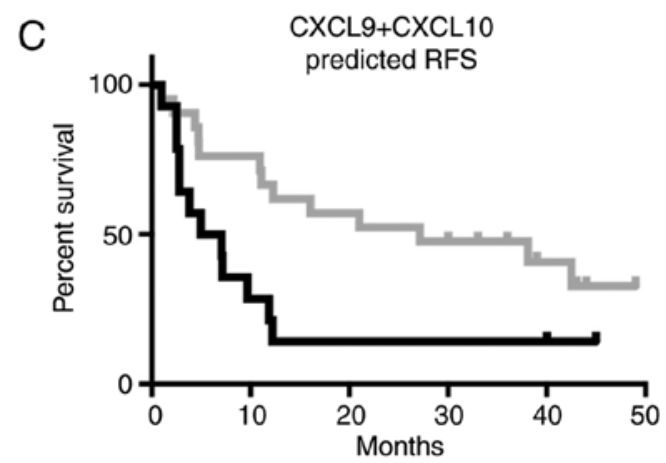

- High risk (mRFS=6.0)
- Low risk (mRFS=27.2)
$\mathrm{P}=0.0183$

D CXCL9+CXCL10

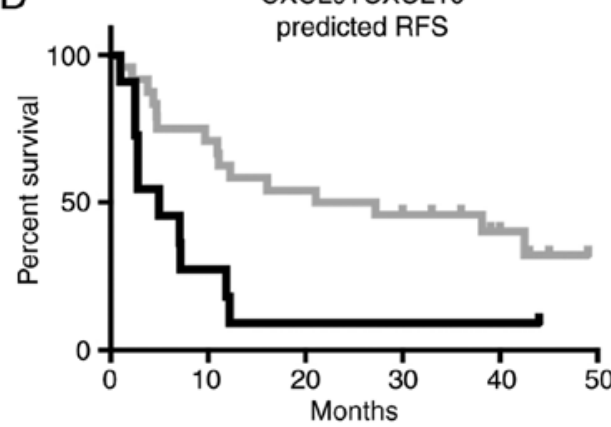

- High risk (mRFS=5.0)

- Low risk (mRFS=24.1) $\mathrm{P}=0.0086$
B

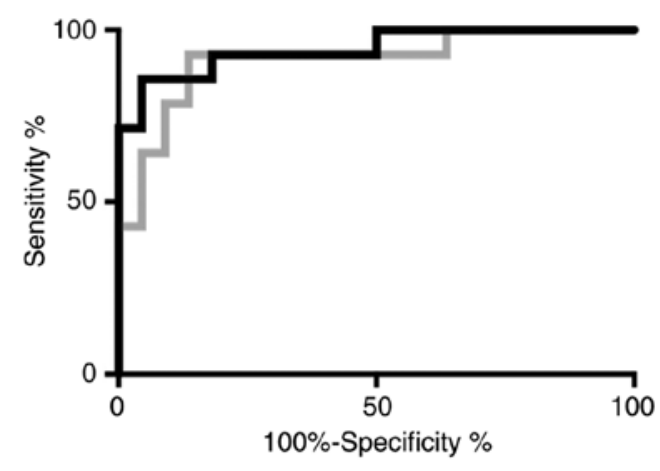

- CXCL9+CXCL10 (AUC=0.945)

- CXCL9 (AUC=0.912)

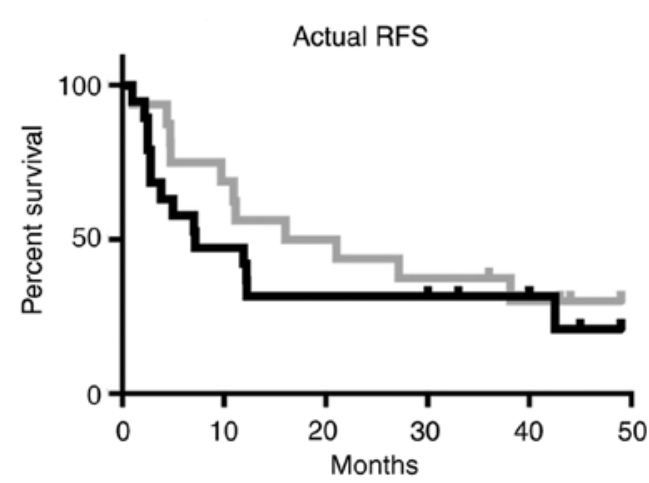

LInflamed (mRFS=7.2)

- Non-inflamed (mRFS=18.6) $p=0.4102$

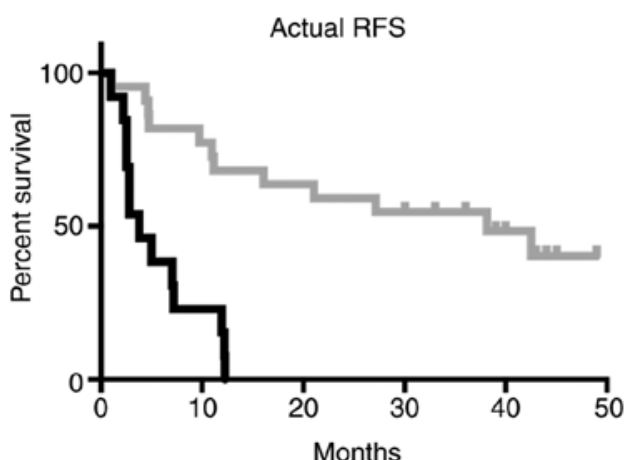

ـInflamed $\mathrm{PFI}<12$ months (mRFS=3.8)

- Rest (mRFS=38.1) $\mathrm{P}<0.0001$

Figure 5. Performance and prognostic impact of CXCL9+CXCL10. ROC curves of CXCL9+CXCL10 and CXCL9 alone in detecting (A) inflamed or (B) inflamed with PFI $<12$ months patients. Survival estimates for patients with the high or low probability of immune tumor infiltration as predicted with CXCL9+CXCL10, compared to actual survival in (C) inflamed vs. non-inflamed patients or (D) inflamed PFI $<12$ months vs. remaining patients. mRFS was calculated from Kaplan Meier survival curves. Survival curves were compared with log-rank test. CCL, C-C motif chemokine ligand; CXCL, C-X-C motif chemokine ligand; AUC, area under curve; RFS, recurrence-free survival; mRFS, median RFS; PFI, platinum-free interval.

again had the most promising characteristics, reaching $86 \%$ sensitivity and $96 \%$ specificity. This combination had good positive and excellent negative clinical utility. Although AUC of CXCL9 alone was 0.912, the combination with CXCL10 performed even better with AUC of 0.945 (Fig. 5C). Patients with high preoperative CXCL9+CXCL10 levels were likely to belong to the inflamed group and recur within 12 months after completion of chemotherapy (Fig. 5D).

\section{Discussion}

Multidirectional cross-talk between various cell types in the tumor microenvironment and recruitment of immune cells is often mediated by the secretion of chemotactic factors (20). Chemokine signals may exhibit pleiotropic effects that go beyond their originally described function as chemoattractants, and can directly and indirectly participate in shaping the 
immune contexture of the tumor (29). Studies demonstrated that the signaling components and metabolites of the tumor microenvironment can gain access to the bloodstream $(21,30)$. Therefore, measuring chemokines in serum of patients with cancer presents as a relevant and convenient approach for identification of novel biomarkers, which may be beneficial for the personalized management of complex diseases, such as recurrent ovarian cancer.

In this study, CC and CXC family chemokines were differentially expressed in sera of patients with HGSOC. Their levels, negatively associated with RFS, reflect the corresponding intratumoral gene and protein expression. A combination of circulating preoperative CCL4 + CXCL1 + CCL20 chemokines was associated with shorter RFS and OS. The immune contexture of tumors was also highlighted and it was proposed that the CXCL9+CXCL10 combination may be applicable for detecting immune-infiltrated tumors, including those which are more likely to recur within 12 months after completion of first-line chemotherapy.

Resistance to platinum-based chemotherapy remains the major cause of HGSOC recurrence. The second-line chemotherapy regimen decision is usually made according to the duration of PFI (5). There are no clinically useful biomarkers to predict the chemotherapy outcome ahead of treatment. As OC is often driven by somatic and germline mutations, attempts to classify tumors as platinum-sensitive or platinum-resistant have typically focused on tumor gene expression profiling and resulted in multiple predictive gene expression algorithms (31-33). Among circulating proteins, the classical OC marker CA-125 was previously demonstrated to be a positive response predictor if present at lower levels at the time of diagnosis (34); however, other studies contradict this finding (35) or emphasize another OC marker, HE4, as more specific in preoperative prediction of platinum sensitivity $(36,37)$.

The development of chemoresistance is a complex process, depending on multiple factors, including intrinsic genetic and epigenetic alterations, cell metabolism, and even the tumor immune infiltration $(38,39)$. The idea that the elements of host immunity may contribute to responsiveness and resistance to chemotherapy suggests the rationale for systemic analysis of soluble mediators. For example, platinum-based drugs and mitotic inhibitors are able to induce nuclear factor $\kappa \mathrm{B}$ (NFKB)-mediated chemoresistance in cancer cells, which relies on the production of proinflammatory chemokines $(40,41)$. We hypothesized that recurrence-prone patients may already exhibit altered levels of serum chemokines at diagnosis. Increased levels of CCL4, CXCL1 and CCL20 chemokines were detected in patients that later experienced disease recurrence. These cytokines act in multiple ways during tumor development and response to treatment. Increased levels of circulating CXCL1 in ovarian carcinomas compared with benign pelvic masses imply its potential role as a marker in early OC detection (42), which may be attributed to its ability to induce the proliferation epithelial $\mathrm{OC}$ cells by transactivation of epidermal growth factor (EGF) receptor and induction of mitogen-activated protein kinase signaling, as demonstrated in vitro (43). CXCL1 participates in an endothelial-carcinoma-myeloid signaling network through its ability to recruit neutrophils/myeloid-derived suppressor cells that promote angiogenesis in vivo and promote cancer cell survival (44).
Chemotherapy-induced tumor necrosis factor- $\alpha$ (TNF- $\alpha$ ) increases the expression of CXCL1, amplifying the signaling loop and causing NFKB-mediated chemoresistance (45). CCL20, similarly to CXCL1, is also expressed in response to EGF and TNF- $\alpha$ (46), and acts pro-metastatically, supporting proliferation, migration and adhesion of tumor cells $(47,48)$. Additionally, CCL20 recruits CD34+-derived dendritic cells and Tregs $(49,50)$. An interesting mechanism was proposed in a study of esophageal squamous cell carcinoma, which suggested that CCL4 and CCL20 recruit functionally different Tymphocyte subsets, cytotoxic T lymphocytes (CTLs) and Tregs, respectively. A high level of CCL20 was associated with worse prognosis, whereas increased CCL4 was associated with better OS (51). Correlation between increased intratumoral CCL4 and $\mathrm{CD}^{+}$TILs was also reported in OC (52). Nevertheless, the present study identified a negative association between cumulative preoperative circulating CCL4 + CXCL1 + CCL20 levels and response to chemotherapy, which may suggest the presence of multiple immune milieu-driven chemoresistance mechanisms in HGSOC.

The discovery of an immunoreactive molecular subtype of HGSOC underlined the complexity of the ovarian tumor microenvironment (TME), indicating that immune effector and inhibitory molecules are expressed in tumors. The presence of CXCL9 and CXCL10 is another characteristic of immunoreactive subtypes $(7,8)$. Together with their receptor $\mathrm{C}-\mathrm{X}-\mathrm{C}$ motif chemokine receptor 3 , these cytokines can indirectly act as both tumor-suppressing and tumor-promoting factors depending on their source. CXCL9 and CXCL10 recruit T and B lymphocytes, natural killer (NK) cells and NK T cells (53). However, their prognostic impact is rather contradictory. We propose the circulating preoperative CXCL9+CXCL10 chemokine classifier as an auxiliary marker for microinvasive discrimination of patients with stronger immune infiltration in tumors. Notably, in the cohort of the present study, there was a negative association between systemic CXCL9+CXCL10 and RFS (but not OS), which contradicts reports linking intratumoral CXCL9 and CXCL10 with better OS in HGSOC to some extent $(13,54)$. However, the levels of these chemokines were also demonstrated to be associated with worse prognosis in several other cancer types (55-60). The complexity of surrounding TME can explain the differences in observed outcomes, as the tumor-dependent factors can shift the microenvironment from immune-activating to immune-suppressing. Additionally, CXCL9 and CXCL10 chemokines can recruit both CTLs and Tregs in ovarian cancer, as demonstrated in vitro and in murine models $(61,62)$, and the CTL/Treg ratio can also determine the prognosis of patients with OC (63). In the present study, elevated circulating chemokine levels reflected the overall $\mathrm{T}$ lymphocyte infiltration. However, gene expression analysis revealed the presence of antitumoral [increased expression of CD8, interferon $\gamma$ (IFN $\gamma)$, granzymes, eomesodermin] and immunosuppressive [increased expression of forkhead box P3, cytotoxic T-lymphocyte associated protein 4 (CTLA-4), PD-L1, interleukin-10 (IL-10), CD163] T cell-associated processes in inflamed tumors. These findings suggest the presence of CTLs and Tregs in the same tumor. Another reason for the contradictory prognostic role of CXCL9 and CXCL10 are their splice variants, which can act antagonistically, as previously reported in hepatocellular carcinoma and HGSOC (64-66). 
Discovery of the immunosuppressive mechanisms of OC encourages the development of novel treatment strategies. The goal of immunotherapy is to generate long-term protective $T$ cell immunity to promote tumor eradication. The cancer-immunity cycle summarizes the stepwise processes required to yield an anticancer $\mathrm{T}$ cell response. One of the crucial stages, trafficking and retaining of effector $\mathrm{T}$ cells in the tumor, is mediated by CXCL9 and CXCL10 (67). Furthermore, the expression of these cytokines, together with IFN $\gamma$ and granzymes, is strongly correlated with the expression of PD-L1 tumors, suggesting the potential benefit of checkpoint blockade (68). Successful melanoma and renal cell carcinoma clinical trials reported the association of intratumoral CXCL9 and CXCL10 in TILs with clinical benefit from adoptive T cell therapy (69), ipilimumab (70,71) and pembrolizumab (72), as well as the increase of circulating CXCL9 and CXCL10 during treatment with nivolumab $(73,74)$. The early phase checkpoint inhibitor trials in OC demonstrated a durable antitumor response in some patients [reviewed in (75)]. The mRNA expression of CTLA-4, PD-L1, IFN $\gamma$, granzyme B and CXCL10 were also increased in immune-infiltrated tumors of patients, characterized by the increased serum CXCL9+CXCL10 level. The relatively short RFS of these patients indicates them as potential candidates for immunotherapy. Thus far, the results of checkpoint inhibition in $\mathrm{OC}$ are promising, taking into consideration the poor sensitivity of platinum-resistant HGSOC to other conventional chemotherapy agents $(75,76)$. Therefore, modulation of TME in patients selected with the help of accurate predictive biomarkers may be an encouraging means for improving OC management and survival. A recent study demonstrated that the expression of CXCL9 and CXCL10, and other genes of IFN $\gamma$ pathway, can be stimulated by chemotherapeutic agents, suggesting that chemotherapy may benefit patients with non-inflamed HGSOC tumors (13). Such pre-treatment may therefore be advantageous for $\mathrm{T}$ cell recruitment and sensitizing tumors to immune modulation. However, the presence of potentially recruited Tregs has to be acknowledged too, as they may impact the choice and strategy of further immunotherapy treatment.

The unsatisfactory clinical outcome of patients with advanced HGSOC urges the search for novel prognostic and predictive biomarkers, and therefore, dictated the exploratory nature of this study. The systemic preoperative chemokine profile was evaluated in patients with $\mathrm{OC}$ and associated them with the immune infiltration of tumors and response to treatment. There are limitations caused by the relatively small sample size and homogeneous ethnicity of the participants in the present study. Also, the study focused on the pre-operative chemokine level measurements and did not follow their dynamics during the primary chemotherapy, recurrence-free period and at the point of recurrence. Longitudinal assessment may provide more a accurate serum chemokine profile in response to treatment, as demonstrated by Coosemans et al (77), which reported the significant decrease in levels of metabolites, including CCL2, IL-10, vascular endothelial growth factor and transforming growth factor- $\beta$, following debulking surgery and paclitaxel/carboplatin-based chemotherapy. Nevertheless, this study provides primary evidence for the combined use of circulating CCL4, CXCL1 and CCL20 for predicting recurrence, and CXCL9 and CXCL10 for detecting immune-infiltrated HGSOC at diagnosis, prior to of treatment. Further validations on a larger scale are required to confirm that these chemokine combinations successfully model the outcome in other patient populations. Additionally, determining the exact source and function of these chemokines in OC setting is necessary for dissecting and targeting the tumor microenvironment.

In conclusion, the findings of the present study demonstrate that preoperative levels of serum chemokines vary among patients with advanced HGSOC. Although the predictive value of single chemokines is modest, their combinations have a potential prognostic use. The results suggest that an increased concentration of circulating CCL4 + CXCL1 + CCL20 in patients with OC serum is associated with shorter RFS and OS. Furthermore, the elevated levels of CXCL9+CXCL10 mark immune infiltrated tumors, and reflect the increased intratumoral gene and protein expression of other inflammatory chemokines. Despite the discussed limitations and descriptive nature of this study, the findings provide background for further investigations of the clinical performance of multiple chemokine combinations as patients stratification tools for improved HGSOC management.

\section{Acknowledgements}

Not applicable.

\section{Funding}

No funding was received.

\section{Availability of data and materials}

The datasets and analyses of this study are available upon request from the corresponding author.

\section{Authors' contributions}

$\mathrm{AM}, \mathrm{MS}$, JAK and VP contributed equally to the conception and design of this study. BI selected the patients and provided their follow-up clinical information. AM, GS, KZ, SG and ND processed the patient samples and performed the experimental procedures. AB performed the histological tumor examination. GL contributed to statistical data analysis. AM interpreted the data and wrote the manuscript. MS, JAK and VP critically revised the manuscript for intellectual content. All authors read and approved the final manuscript.

\section{Ethics approval and consent to participate}

This study was approved by Lithuanian Bioethics Committee (approval no. 158200-06-500-147). All patients signed an informed consent form.

\section{Patient consent for publication}

All patients signed an informed consent form allowing for publication of depersonalized data.

\section{Competing interests}

The authors declare that they have no competing interests. 


\section{References}

1. Sant M, Allemani C, Santaquilani M, Knijn A, Marchesi F and Capocaccia R; EUROCARE Working Group: EUROCARE-4 Survival of cancer patients diagnosed in 1995-1999. Results and commentary. Eur J Cancer 45: 931-991, 2009.

2. Sant M, Chirlaque Lopez MD, Agresti R, Sánchez Perez MJ, Holleczek B, Bielska-Lasota M, Dimitrova N, Innos K, Katalinic A, Langseth $\mathrm{H}$, et al: Survival of women with cancers of breast and genital organs in Europe 1999-2007: Results of the EUROCARE-5 study. Eur J Cancer 51: 2191-2205, 2015.

3. Bowtell DD, Böhm S, Ahmed AA, Aspuria PJ, Bast R, Beral V, Berek JS, Birrer MJ, Blagden S, Bookman MA, et al: Rethinking ovarian cancer II: Reducing mortality from high-grade serous ovarian cancer. Nat Rev Cancer 15: 668-679, 2015.

4. Matulonis UA, Sood AK, Fallowfield L, Howitt BE, Sehouli J and Karlan BY: Ovarian cancer. Nat Rev Dis Primers 2: 16061, 2016.

5. Friedlander M,TrimbleE,Tinker A,Alberts D, Avall-LundquistE, Brady M, Harter P, Pignata S, Pujade-Lauraine E, Sehouli J, et al Clinical trials in recurrent ovarian cancer. Int $\mathrm{J}$ Gynecol Cancer 21: 771-775, 2011.

6. Alvarez RD, Matulonis UA, Herzog TJ, Coleman RL, Monk BJ and Markman M: Moving beyond the platinum sensitive/resistant paradigm for patients with recurrent ovarian cancer. Gynecol Oncol 141: 405-409, 2016.

7. Bell D, Berchuck A, Birrer M, Chien J, Cramer D, Dao F, Dhir R, DiSala P, Gabra H, Glenn P, et al: Integrated genomic analyses of ovarian carcinoma. Nature 474: 609-615, 2011.

8. Tothill RW, Tinker AV, George J, Brown R, Fox SB, Lade S Johnson DS, Trivett MK, Etemadmoghadam D, Locandro B, et al: Novel molecular subtypes of serous and endometrioid ovarian cancer linked to clinical outcome. Clin Cancer Res 14: 5198-5208, 2008

9. Verhaak RG, Tamayo P, Yang JY, Hubbard D, Zhang H, Creighton CJ, Fereday S, Lawrence M, Carter SL, Mermel CH, et al: Prognostically relevant gene signatures of high-grade serous ovarian carcinoma. J Clin Invest 123: 517-525, 2013.

10. Konecny GE, Wang C, Hamidi H, Winterhoff B, Kalli KR, Dering J, Ginther C, Chen HW, Dowdy S, Cliby W, et al: Prognostic and therapeutic relevance of molecular subtypes in high-grade serous ovarian cancer. J Natl Cancer Inst 106: pii: dju249, 2014.

11. Nelson BH: New insights into tumor immunity revealed by the unique genetic and genomic aspects of ovarian cancer. Curr Opin Immunol 33: 93-100, 2015

12. Hwang WT, Adams SF, Tahirovic E, Hagemann IS and Coukos G: Prognostic significance of tumor-infiltrating $\mathrm{T}$ cells in ovarian cancer: A meta-analysis. Gynecol Oncol 124: 192-198, 2012.

13. Hao D, Liu J, Chen M, Li J, Wang L, Li X, Zhao Q and Di LJ: Immunogenomic analyses of advanced serous ovarian cancer reveal immune score is a prognostic factor and an indicator of chemosensitivity. Clin Cancer Res 24: 3560-3571, 2018.

14. Wang W, Kryczek I, Dostál L, Lin H, Tan L, Zhao L, Lu F, Wei S, Maj T, Peng D, et al: Effector T cells abrogate Stroma-mediated chemoresistance in ovarian cancer. Cell 165: 1092-1105, 2016.

15. Xu S, Tao Z, Hai B, Liang H, Shi Y, Wang T, Song W, Chen Y, OuYang J, Chen J, et al: miR-424(322) reverses chemoresistance via T-cell immune response activation by blocking the PD-L1 immune checkpoint. Nat Commun 7: 11406, 2016.

16. Rabinovich GA, Gabrilovich D and Sotomayor EM: Immunosuppressive strategies that are mediated by tumor cells. Annu Rev Immunol 25: 267-296, 2007.

17. Castells M, Thibault B, Delord JP and Couderc B: Implication of tumor microenvironment in chemoresistance: Tumor-associated stromal cells protect tumor cells from cell death. Int J Mol Sci 13: 9545-9571, 2012

18. Baghdadi M,Wada H,Nakanishi S, Abe H,Han N,WiraE,Endo D, Watari H, Sakuragi N, Hida Y, et al: Chemotherapy-induced IL34 enhances immunosuppression by tumor-associated macrophages and mediates survival of chemoresistant lung cancer cells. Cancer Res 76: 6030-6042, 2016.

19. Takeuchi S, Baghdadi M, Tsuchikawa T, Wada H, Nakamura T, Abe H, Nakanishi S, Usui Y, Higuchi K, Takahashi M, et al: Chemotherapy-derived inflammatory responses accelerate the formation of immunosuppressive myeloid cells in the tissue microenvironment of human pancreatic cancer. Cancer Res 75 2629-2640, 2015.

20. Mukaida N, Sasaki S and Baba T: Chemokines in cancer development and progression and their potential as targeting molecules for cancer treatment. Mediators Inflamm 2014: 170381, 2014.
21. Gnjatic S, Bronte V, Brunet LR, Butler MO, Disis ML, Galon J, Hakansson LG, Hanks BA, Karanikas V, Khleif SN, et al: Identifying baseline immune-related biomarkers to predict clinical outcome of immunotherapy. J Immunother Cancer 5: 44, 2017.

22. Yang WL, Lu Z and Bast RC Jr: The role of biomarkers in the management of epithelial ovarian cancer. Expert Rev Mol Diagn 17: 577-591, 2017.

23. Prat $\mathrm{J}$ and FIGO Committee on Gynecologic Oncology: Abridged republication of FIGO's staging classification for cancer of the ovary, fallopian tube, and peritoneum. Cancer 121: 3452-3454, 2015.

24. Livak KJ and Schmittgen TD: Analysis of relative gene expression data using real-time quantitative PCR and the $2^{-\Delta \Delta C_{\mathrm{T}}}$ method. Methods 25: 402-408, 2001

25. Pfaffl MW: A new mathematical model for relative quantification in real-time RT-PCR. Nucleic Acids Res 29: e45, 2001.

26. Shimizu Y, Kamoi S, Amada S, Akiyama F and Silverberg SG: Toward the development of a universal grading system for ovarian epithelial carcinoma: Testing of a proposed system in a series of 461 patients with uniform treatment and follow-up. Cancer 82: 893-901, 1988.

27. Kaku T, Ogawa S, Kawano Y, Ohishi Y, Kobayashi H, Hirakawa T and Nakano H: Histological classification of ovarian cancer. Med Electron Microsc 36: 9-17, 2003.

28. Mitchell AJ: Sensitivity x PPV is a recognized test called the clinical utility index (CUI+). Eur J Epidemiol 26: 251-252, 2011.

29. Charo IF and Ransohoff RM: The many roles of chemokines and chemokine receptors in inflammation. N Engl J Med 354: 610-621, 2006.

30. Pitteri SJ, Kelly-Spratt KS, Gurley KE, Kennedy J, Buson TB, Chin A, Wang $\mathrm{H}$, Zhang Q, Wong $\mathrm{CH}$, Chodosh LA, et al: Tumor microenvironment-derived proteins dominate the plasma proteome response during breast cancer induction and progression. Cancer Res 71: 5090-5100, 2011

31. Gevaert O, De Smet F, Van Gorp T, Pochet N, Engelen K, Amant F, De Moor B, Timmerman D and Vergote I: Expression profiling to predict the clinical behaviour of ovarian cancer fails independent evaluation. BMC Cancer 8: 18, 2008.

32. Helleman J, Jansen MP, Span PN, van Staveren IL, Massuger LF, Meijer-van Gelder ME, Sweep FC, Ewing PE, van der Burg ME, Stoter G, et al: Molecular profiling of platinum resistant ovarian cancer. Int J Cancer 118: 1963-1971, 2006.

33. Gonzalez Bosquet J, Newtson AM, Chung RK, Thiel KW, Ginader T, Goodheart MJ, Leslie KK and Smith BJ: Prediction of chemo-response in serous ovarian cancer. Mol Cancer 15: 66, 2016.

34. Chung RK, Newtson AM, Mott SL and Gonzalez Bosquet J: Clinicopathological predictors of responsiveness in epithelial ovarian cancer: A preliminary institutional study. Proc Obstet Gynecol 5: 6, 2015.

35. Masoumi-Moghaddam S, Amini A, Wei AQ, Robertson G and Morris DL: Vascular endothelial growth factor expression correlates with serum CA125 and represents a useful tool in prediction of refractoriness to platinum-based chemotherapy and ascites formation in epithelial ovarian cancer. Oncotarget 6: 28491-28501, 2015

36. Angioli R, Capriglione S, Aloisi A, Guzzo F, Luvero D, Miranda A, Damiani P, Montera R, Terranova C and Plotti F: Can HE4 predict platinum response during first-line chemotherapy in ovarian cancer? Tumor Biol 35: 7009-7015, 2014.

37. Chudecka-Głaz A, Cymbaluk-Płoska A, Wężowska $M$ and Menkiszak J: Could HE4 level measurements during first-line chemotherapy predict response to treatment among ovarian cancer patients? PLoS One 13: e0194270, 2018.

38. Pogge von Strandmann E, Reinartz S, Wager U and Müller R: Tumor-host cell interactions in ovarian cancer: Pathways to therapy failure. Trends Cancer 3: 137-148, 2017.

39. Senthebane DA, Rowe A, Thomford NE, Shipanga H, Munro D Mazeedi MAM, Almazyadi HAM, Kallmeyer K, Dandara C, Pepper MS, et al: The role of tumor microenvironment in chemoresistance: To survive, keep your enemies closer. Int J Mol Sci 18: pii: E1586, 2017.

40. de Visser KE and Jonkers J: Towards understanding the role of cancer-associated inflammation in chemoresistance. Curr Pharm Des 15: 1844-1853, 2009.

41. Szajnik M, Szczepanski MJ, Czystowska M, Elishaev E, Mandapathil M, Nowak-Markwitz E, Spaczynski M and Whiteside TL: TLR4 signaling induced by lipopolysaccharide or paclitaxel regulates tumor survival and chemoresistance in ovarian cancer. Oncogene 28: 4353-4363, 2009. 
42. Wang Q, Li D, Zhang W, Tang B, Li OQ and Li L: Evaluation of proteomics-identified CCL18 and CXCL1 as circulating tumor markers for differential diagnosis between ovarian carcinomas and benign pelvic masses. Int J Biol Markers 26: 262-273, 2011.

43. Bolitho C, Hahn MA, Baxter RC and Marsh DJ: The chemokine CXCL1 induces proliferation in epithelial ovarian cancer cells by transactivation of the epidermal growth factor receptor. Endocr Relat Cancer 17: 929-940, 2010.

44. ScapiniP,MoriniM,TecchioC,MinghelliS,DiCarloE,TanghettiE, Albini A, Lowell C, Berrton G, Noonan DM and Cassatella MA: CXCL1/macrophage inflammatory protein-2-induced angiogenesis in vivo is mediated by neutrophil-derived vascular endothelial growth factor-A. J Immunol 172: 5034-5040, 2004.

45. Acharyya S, Oskarsson T, Vanharanta S, Malladi S, Kim J, Morris PG, Manova-Todorova K, Leversha M, Hogg N, Seshan VE, et al: A CXCL1 paracrine network links cancer chemoresistance and metastasis. Cell 150: 165-178, 2012.

46. Son DS, Kabir SM, Dong Y, Lee E and Adunyah SE: Characteristics of chemokine signatures elicited by EGF and TNF in ovarian cancer cells. J Inflamm 10: 25, 2013.

47. Marsigliante S, Vetrugno C and Muscella A: CCL20 induces migration and proliferation on breast epithelial cells. J Cell Physiol 228: 1873-1883, 2013.

48. Beider K, Abraham M, Begin M, Wald H, Weiss ID, Wald O, Pikarsky E, Abramovitch R, Zeira E, Galun E, et al: Interaction between CXCR4 and CCL20 pathways regulates tumor growth. PLoS One 4: e5125, 2009.

49. Greaves DR, Wang W, Dairaghi DJ, Dieu MC, Saint-Vis B, Franz-Bacon K, Rossi D, Caux C, McClanahan T, Gordon S, et al: CCR6, a CC chemokine receptor that interacts with macrophage inflammatory protein $3 \alpha$ and is highly expressed in human dendritic cells. J Exp Med 186: 837-844, 1997.

50. Cook KW, Letley DP, Ingram RJ, Staples E, Skjoldmose H, Atherton JC and Robinson K: CCL20/CCR6-mediated migration of regulatory $\mathrm{T}$ cells to the Helicobacter pylori-infected human gastric mucosa. Gut 63: 1550-1559, 2014

51. Liu JY, Li F, Wang LP, Chen XF, Wang D, Cao L, Ping Y, Zhao S, Li B, Thorne SH, et al: CTL- vs $\mathrm{T}_{\text {re }}$ lymphocyte-attracting chemokines, CCL4 and CCL20, are strong reciprocal predictive markers for survival of patients with oesophageal squamous cell carcinoma. Br J Cancer 113: 747-755, 2015.

52. Zsiros E, Duttagupta P, Dangaj D, Li H, Frank R, Garrabrant T, Hagemann IS, Levine BL, June $\mathrm{CH}$, Zhang L, et al: The ovarian cancer chemokine landscape is conducive to homing of vaccine-primed and $\mathrm{CD} 3 / \mathrm{CD} 28$-costimulated $\mathrm{T}$ cells prepared for adoptive therapy. Clin Cancer Res 21: 2840-2850, 2015.

53. Tokunaga R, Zhang W, Naseem M, Puccini A, Berger MD, Soni S, McSkane M, Baba H and Lenz HJ: CXCL9, CXCL10, CXCL11/CXCR3 axis for immune activation-a target for novel cancer therapy. Cancer Treat Rev 63: 40-47, 2018.

54. Bronger H, Singer J, Windmüller C, Reuning U, Zech D Delbridge C, Dorn J, Kiechle M, Schmalfeldt B, Schmitt M and Avril S: CXCL9 and CXCL10 predict survival and are regulated by cyclooxygenase inhibition in advanced serous ovarian cancer. Br J Cancer 115: 553-563, 2016.

55. Mir MA, Maurer MJ, Ziesmer SC, Slager SL, Habermann T, Macon WR, Link BK, Syrbu S, Witzig T, Friedberg JW, et al: Elevated serum levels of IL-2R, IL-1RA, and CXCL9 are associated with a poor prognosis in follicular lymphoma. Blood 125 : 992-998, 2015

56. Flores RJ, Kelly AJ, Li Y, Nakka M, Barkauskas DA, Krailo M, Wang LL, Perlaky L, Lau CC, Hicks MJ, et al: A novel prognostic model for osteosarcoma using circulating CXCL10 and FLT3LG. Cancer 123: 144-154, 2017.

57. Hong JY, Ryu KJ, Lee JY, Park C, Ko YH, Kim WS and Kim SJ: Serum level of CXCL10 is associated with inflammatory prognostic biomarkers in patients with diffuse large B-cell lymphoma Hematol Oncol 35: 480-486, 2017.

58. Bai M, Chen X and Ba YI: CXCL10/CXCR3 overexpression as a biomarker of poor prognosis in patients with stage II colorectal cancer. Mol Clin Oncol 4: 23-30, 2016.

59. Toiyama Y, Fujikawa H, Kawamura M, Matsushita K, Saigusa S, Tanaka K, Inoue Y, Uchida M, Mohri Y and Kusunoki M. Evaluation of CXCL10 as a novel serum marker for predicting liver metastasis and prognosis in colorectal cancer. Int J Oncol 40: $560-566,2012$
60. Specht K, Harbeck N, Smida J, Annecke K, Reich U, Naehrig J, Langer R, Mages J, Busch R, Kruse E, et al: Expression profiling identifies genes that predict recurrence of breast cancer after adjuvant CMF-based chemotherapy. Breast Cancer Res Treat 118 45-56, 2009.

61. K Au K, Peterson N, Truesdell P, Reid-Schachter G, Khalaj K, Ren R, Francis JA, Graham CH, Craig AW and Koti M: CXCL10 alters the tumour immune microenvironment and disease progression in a syngeneic murine model of high-grade serous ovarian cancer. Gynecol Oncol 145: 436-445, 2017.

62. Redjimi N, Raffin C, Raimbaud I, Pignon P, Matsuzaki J, Odunsi K, Valmori D and Ayyoub M: CXCR $3^{+}$T regulatory cells selectively accumulate in human ovarian carcinomas to limit type I immunity. Cancer Res 72: 4351-4360, 2012.

63. Sato E, Olson SH, Ahn J, Bundy B, Nishikawa H, Qian F Jungbluth AA, Frosina D, Gnjatic S, Ambrosone C, et al: Intraepithelial CD8+ tumor-infiltrating lymphocytes and a high $\mathrm{CD}^{+} /$regulatory $\mathrm{T}$ cell ratio are associated with favorable prognosis in ovarian cancer. Proc Natl Acad Sci USA 102: $18538-18543,2005$.

64. Casrouge A, Decalf J, Ahloulay M, Lababidi C, Mansour H, Vallet-Pichard A, Mallet V, Mottez E, Mapes J, Fontanet A, et al: Evidence for an antagonist form of the chemokine CXCL10 in patients chronically infected with HCV. J Clin Invest 121: 308-317, 2011.

65. Rainczuk A, Rao JR, Gathercole JL, Fairweather NJ, Chu S, Masadah R, Jobling TW, Deb-Choudhury S, Dyer J and Stephens AN: Evidence for the antagonistic form of CXC-motif chemokine CXCL10 in serous epithelial ovarian tumours. Int J Cancer 134: 530-541, 2014.

66. Ding Q, Lu P, Xia Y, Ding S, Fan Y, Li X, Han P, Liu J, Tian D and Liu M: CXCL9: Evidence and contradictions for its role in tumor progression. Cancer Med 5: 3246-3259, 2016.

67. Chen DS and Mellman I: Oncology meets immunology: The cancer-immunity cycle. Immunity 39: 1-10, 2013.

68. Peng W, Liu C, Xu C, Lou Y, Chen J, Yang Y, Yagita H, Overwijk WW, Lizée G, Radvanyi L, et al: PD-1 blockade enhances T-cell migration to tumors by elevating IFN- $\gamma$ inducible chemokines. Cancer Res 72: 5209-5218, 2012

69. Bedognetti D, Spivey TL, Zhao Y, Uccellini L, Tomei S, Dudley ME, Ascierto ML, De Giorgi V, Liu Q, Delogu LG, et al: CXCR3/CCR5 pathways in metastatic melanoma patients treated with adoptive therapy and interleukin-2. Br J Cancer 109: 2412-2423, 2013

70. Harlin H, Meng Y, Peterson AC, Zha Y, Tretiakova M, Slingluff C, McKee $\mathrm{M}$ and Gajewski TF: Chemokine expression in melanoma metastases associated with $\mathrm{CD} 8^{+} \mathrm{T}$-cell recruitment. Cancer Res 69: 3077-3085, 2009.

71. Tarhini AA, Lin Y, Lin HM, Vallabhaneni P, Sander C, LaFramboise $W$ and Hamieh L: Expression profiles of immune-related genes are associated with neoadjuvant ipilimumab clinical benefit. Oncoimmunology 6: e1231291, 2016.

72. Ayers M, Lunceford J, Nebozhyn M, Murphy E, Loboda A, Kaufman DR, Albright A, Cheng JD, Kang SP, Shankaran V, et al: IFN- $\gamma$-related mRNA profile predicts clinical response to PD-1 blockade. J Clin Invest 127: 2930-2940, 2017.

73. Yamazaki N, Kiyohara Y, Uhara H, Iizuka H, Uehara J, Otsuka F, Fujisawa Y, Takenouchi T, Isei T, Iwatsuki K, et al: Cytokine biomarkers to predict antitumor responses to nivolumab suggested in a phase 2 study for advanced melanoma. Cancer Sci 108: 1022-1031, 2017.

74. Choueiri TK, Fishman MN, Escudier B, McDermott DF, Drake CG, Kluger H, Stadler WM, Perez-Gracia JL, McNeel DG, Curti B, et al: Immunomodulatory activity of nivolumab in metastatic renal cell carcinoma. Clin Cancer Res 22: 5461-5471, 2016

75. Hamanishi J, Mandai $\mathbf{M}$ and Konishi I: Immune checkpoint inhibition in ovarian cancer. Int Immunol 28: 339-348, 2016.

76. Hamanishi J, Mandai M, Ikeda T, Minami M, Kawaguchi A, Murayama T, Kanai M, Mori Y, Matsumoto S, Chikuma S, et al: Safety and antitumor activity of anti-PD-1 antibody, nivolumab, in patients with platinum-resistant ovarian cancer. J Clin Oncol 33: 4015-4022, 2015.

77. Coosemans A, Decoene J, Baert T, Laenen A, Kasran A, Verschuere T, Seys S and Vergote I: Immunosuppressive parameters in serum of ovarian cancer patients change during the disease course. Oncoimmunology 5: e1111505, 2016. 